\title{
Commentary: A case for getting bogged down in the details-Post-coronary artery bypass grafting atrial fibrillation
}

\author{
Siamak Mohammadi, MD, FRCSC, and Dimitri Kalavrouziotis, MD, FRCSC
}

\author{
From the Department of Cardiac Surgery, Quebec Heart and Lung Institute, Quebec City, Québec, Canada. \\ Disclosures: Authors have nothing to disclose with regard to commercial support. \\ Received for publication March 5, 2019; accepted for publication March 5, 2019; available ahead of print April \\ $10,2019$. \\ Address for reprints: Siamak Mohammadi, MD, FRCSC, Department of Cardiac Surgery, Quebec Heart and Lung \\ Institute, 2725 Chemin Sainte-Foy, Quebec City, Québec, Canada, G1V 4G5 (E-mail: siamak.mohammadi@ \\ fmed.ulaval.ca). \\ J Thorac Cardiovasc Surg 2020;159:526-502 \\ $0022-5223 / \$ 36.00$ \\ Copyright (c) 2019 by The American Association for Thoracic Surgery \\ https://doi.org/10.1016/j.jtcvs.2019.03.013
}

The prevalence of new-onset postoperative atrial fibrillation (POAF) has not changed during the past few decades, and many studies have been performed to identify patients at highest risk and prophylactic strategies to prevent POAF. ${ }^{1}$ In this issue of the Journal, Bening and colleagues ${ }^{2}$ attempt to determine a "unifying theory" of POAF on the basis of a pathophysiologic model that uses serum and tissue biomarkers, contractile characteristics of left and right atrial appendages removed at surgery, and clinical and echocardiographic variables, between patients who had POAF develop after elective coronary artery bypass grafting (CABG) and those who did not. Two hundred twenty-nine patients without a history of atrial fibrillation were recruited prospectively, and $16.8 \%$ had POAF develop. In multivariate analysis, the independent risk factors for POAF after CABG were (1) advanced age, (2) higher left and right atrial areas on 2-dimensional echocardiography, (3) lower tricuspid annular plane systolic excursion, and, finally, (4) reduced left atrial myofilament contractile force in an ex vivo analysis of human tissue. Other inflammatory and fibrotic biomarkers were not associated with POAF.

That the typical patient who had POAF develop after CABG was an elderly hypertensive patient with renal failure and probable left ventricular diastolic dysfunction should come as no surprise. Advanced age is by far the number-one determinant of POAF in a CABG population, ${ }^{3}$ and it seems to be at the core of the pathogenetic processes involved in POAF, including loss of myocardial fibers, increased interstitial collagen deposition, and atrial remodeling and enlargement. ${ }^{4}$ To what extent the loss of atrial contractile function occurs independently of age in influencing the risk of POAF after CABG cannot be clearly established by the study of Bening and colleagues. ${ }^{2}$ Statistical interactions and collinearity weaken purported independent associations in multivariate analyses; these were not clearly reported. Bening and colleagues ${ }^{2}$ also identified left atrial size as an independent risk factor for POAF. The clinical

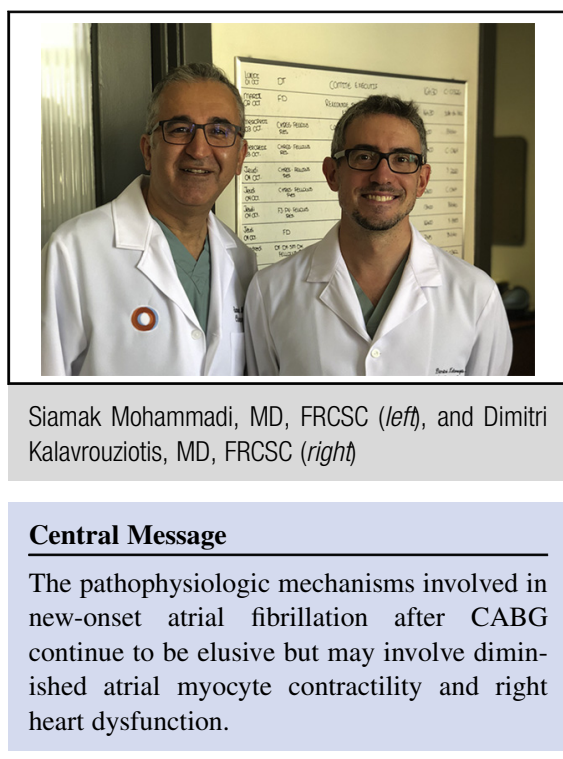

See Article page 515 .

prognostic value of left atrial enlargement for adverse cardiovascular outcomes is not new. ${ }^{5}$ Quantifying left atrial size is difficult, however, and current recommendations of the American Society of Echocardiography ${ }^{6}$ suggest that left atrial volume should be indexed to body surface area as a more accurate predictor of clinical outcome than simple, unindexed 2-dimensional atrial area measurements, as used in the study of Bening and colleagues. ${ }^{2}$

One interesting and novel finding of the study of Bening and colleagues ${ }^{2}$ was the predictive value of right atrial enlargement and lower tricuspid annular plane systolic excursion in development of POAF. Bening and colleagues ${ }^{2}$ did not define a cutoff reference value of atrial area (either left or right), which may serve as a trigger to consider preoperative preventative pharmacologic therapies to decrease the risk of POAF after $\mathrm{CABG}$.

The demonstration of decreased responsiveness of atrial myocytes to calcium from excised left and right atrial appendages among patients who had POAF develop after CABG versus those who did not is certainly compelling, because it suggests the presence of a long-standing substrate for the subsequent development of POAF in patients without preexisting AF. The use of this criterion as a riskstratifying tool to predict POAF after CABG is difficult, however, and even impossible in everyday practice. The 
central role of atrial functional remodeling in the pathogenesis of atrial fibrillation is well known. ${ }^{7}$ Left atrial strain analysis and volumetric parameters (eg, left atrial ejection fraction) with advanced echocardiographic or cardiac magnetic resonance imaging techniques are future modalities to explore and may help to identify patients at risk for POAF.

The study of Bening and colleagues ${ }^{2}$ supports the contention that, in addition to the usual clinical risk factors, atrial size measurements and functional assessment may become promising tools in risk stratification and preventive decision making for patients undergoing CABG. Further studies are needed to identify precise cutoff values of left and right atrial size and function adjusted to the patient's age and body surface area in predicting POAF, which may allow the administration of individualized prophylactic measures, including pharmacologic therapy ${ }^{3}$ or perioperative atrial override pacing. ${ }^{8}$

\section{References}

1. Greenberg JW, Lancaster TS, Schuessler RB, Melby SJ. Postoperative atrial fibrillation following cardiac surgery: a persistent complication. Eur J Cardiothorac Surg. 2017;52:665-72.
2. Bening C, Mazalu EA, Yaqub J, Alhussini K, Glanowski M, Kottmann T, et al. Atrial contractility and fibrotic biomarkers are associated with atrial fibrillation after elective coronary artery bypass grafting. J Thorac Cardiovasc Surg. 2020;159: 515-23.

3. Echahidi N, Pibarot P, O'Hara G, Mathieu P. Mechanisms, prevention, and treatment of atrial fibrillation after cardiac surgery. J Am Coll Cardiol. 2008;51: 793-801.

4. Shen J, Lall S, Zheng V, Buckley P, Damiano RJ Jr, Schuessler RB. The persistent problem of new-onset postoperative atrial fibrillation: a singleinstitution experience over two decades. J Thorac Cardiovasc Surg. 2011; 141:559-70.

5. Hoit BD. Left atrial size and function: role in prognosis. J Am Coll Cardiol. 2014; 63:493-505.

6. Lang RM, Bierig M, Devereux RB, Flachskampf FA, Foster E, Pellikka P, et al; Chamber Quantification Writing Group, American Society of Echocardiography's Guidelines and Standards Committee, European Association of Echocardiography. Recommendations for chamber quantification: a report from the American Society of Echocardiography's Guidelines and Standards Committee and the Chamber Quantification Writing Group developed in conjunction with the European Association of Echocardiography, a branch of the European Society of Cardiology. J Am Soc Echocardiogr. 2005;18:1440-63.

7. Thomas L, Abhayaratna WP. Left atrial reverse remodeling: mechanisms, evaluation, and clinical significance. JACC Cardiovasc Imaging. 2017;10: $65-77$.

8. Blommaert D, Gonzalez M, Mucumbitsi J, Gurné O, Evrard P, Buche M, et al. Effective prevention of atrial fibrillation by continuous atrial overdrive pacing after coronary artery bypass surgery. J Am Coll Cardiol. 2000;35: 1411-5. 